\title{
CARACTERIZAÇĀO DE CEPAS DE P. FALCIPARUM COLETADAS DE PACIENTES RECRUDESCENTES
}

Virgilio E. do ROSARIo (1) (*), Álvaro A. COUTo (1), Marco A. SANTOS (1) e Josê Maria de SouZa (2)

\section{R E S U M O}

A caracterização de cepas de $\mathbf{P}$. falciparum coletadas de três pacientes internados no Centro de Provas Clínico-Farmacológicas do Hospital Barros Barreto (Belém, PA), através da eletroforese em acetato de celulose para duas enzimas (GPI e ADA), assim como através de testes de sensibilidade para duas drogas, cloroquina e mefloquina, permitiram observações da variação dos tipos enzimáticos em um dos pacientes e modificação no nivel de sensibilidade à cloroquina em outro, tanto em amostras obtidas da infecção original, como em cada uma das recrudescências.

\section{N T R O D U C $\mathbf{A} \tilde{A} O$}

A existência de populações de parasitas, diferenciadas através das usuiais técnicas de caracterização até hoje utilizadas - testes de resistência à drogas, perfil isoenzimático, diversidade antigênica, maior ou menor capacidade adaptativa às condições de cultivo, entre outräs - confirma que, ao se comparar amostras coletadas em regiões diferentes, ou até mesmo numa mesma área, estas populações pođem ser đistintas (THAITHONG 5; ROSÂRIO ${ }^{2}$ ).

O programa de caracterização de cepas estabelecido na Unidade de Estudos da Malária (Instituto Evandro Chagas - FSESP), até agora baseou seus estudos na análise das amostras coletadas de pacientes oriundos de localidades distintas da Região Amazônica. Estas amostras, também denominadas cepas, referem-se a populações de parasitas de Plasmodium falciparum obtidas em uma única coleta, de um deter. minado paciente ${ }^{2}$. Nossos estudos também já comprovaram, utilizanđo-se a técnica de eletroforese em acetato de celulose, a ocorrência de populações diferentes coexistentes em amostra de um único paciente ${ }^{1}$. Tal observação, sugeriu que uma nova linha de pesquisa fosse realizada; obtendo amostras para cultivo contínuo e caracterização antes e após tratamento (paciente recrudescente) seria possível observar-se o grau de identidade entre ambas as populações de parasitas, coletados de um mesmo paciente. Usualmente sempre se considerou que o paciente portador de malária, resistente a uma dada medicação, fosse portador de uma população homogênea de parasitas.

Demonstrando-se que, em um paciente que recrudesceu, a população de parasitas que sobreviveu a uma determinada međicação é diferente da população original, cremos que maior atenção deverá ser atribuída a estes casos clínicos.

Acreditamos que este estudo possa auxiliar a interpretação clínica de alguns casos de resistência a determinado tratamento, como também oferecer informações relevantes a futu ros programas de controle, baseado em medicação profilática.

(1) Unidade de Estudos da Malária, Instituto Evandro Chagas (FSESP) - Belém, PA

(2) Centro de Provas Clínico-Farmacológicas, Hospital Barros Barreto - Projeto Mefloquina, Belém, PA

${ }^{*}$ Atualmente fixado na Univ. of North Carolina-Parasitology Chapel Hill - USA 
ROSARIO, V. E. do; COUTO, A. A.; SANTOS, M. A. \& SOUZA, J. M. de - Caracterização de cepas de P. falciparum co letadas de pacientes recrudescentes. Rev. Ins!. Mrail. Hop. São Paulo 27:?74-278, 1985.

\section{MATERIAIS E MÉTODOS}

As amostras analisadas neste trabalho foram coletadas de três pacientes (FRL; PPS e APJ), internados no Centro de Provas ClínicoFarmacológicas do Hospital Barros Barreto Projeto Mefloquina (Belém, PA) ${ }^{4}$, os quais apresentaram recrudescência após um ou mais tratamentos. Não foi registrada a ocorrência de vômito nos dois primeiros pacientes (FRL e PPS), o que só sucedeu no terceiro (APJ), que vomitou 25 minutos após o tratamento, voltando a vomitar mais duas vezes dentro da primeira hora (*). O material coletado foi cultivado pela técnica de TRAGER \& JENSEN ${ }^{6}$.

O paciente (FRL), internado a 06.07 .83 , foi tratado com o esquema sulfato de quinino 0,6 $\mathrm{g}$ de $8 / 8$ horas durante 3 dias, associado a pirimetamina-sulfadoxina 3 comprimidos, dose única, 4 horas após a primeira dose de sulfato de quinino. Após negativar, veio recrudescer a 21 do mesmo mês, ainda hospitalizado, e foi medicado com 3 comprimidos de pirimetamina-sulfadoxina, sem sucesso, obrigando-se a um novo tratamento, desta vez com amodiaquina $10 \mathrm{mg}$ / kg no primeiro dia, $7,5 \mathrm{mg} / \mathrm{kg}$ no segundo e $7,5 \mathrm{mg} / \mathrm{kg}$ no terceiro dia, o que também não produziu efeito curativo. Finalmente, em 16.08.83 foi tratado com sulfametrol $0,8 \mathrm{~g}+$ trimetropim $0,16 \mathrm{~g}$ de $12 / 12$ horas durante 5 dias, negativando em 20.08 e recebendo alta em 25.08 .83 ainda negativo, data do último controle. As amostras coletadas receberam os números: $70 /$ $83,84 / 83,87 / 83$ e $97 / 83$.

O paciente (PPS) foi internado a 29.07.83 tratado com cloridrato de quinino $0,53 \mathrm{~g}$ de $8 / 8$ horas durante 3 dias, associado a pirimetaminasulfadoxina 3 comprimidos, dose única, em 04 . 08.83. Após negativar, recrudesceu em 11.08. 83 e foi medicađo, após coleta de amostra para cultivo, em $15.08 .83 \mathrm{com}$ amodiaquina $10 \mathrm{mg} / \mathrm{kg}$ no primeiro dia, $7,5 \mathrm{mg} / \mathrm{kg}$ no segundo dia $\mathrm{e}$ $7,5 \mathrm{mg} / \mathrm{kg}$ no terceiro dia, sem sucesso. Recebeu um terceiro tratamento em 25.08.83 à base de sulfato de quinino $0,6 \mathrm{~g}$ de $8 / 8$ horas, associado à cloridrato de tetraciclina $0,25 \mathrm{~g}$ de $6 / 6$ horas durante 10 dias, negativando e recebendo alta em 03.09.83. Fez controle ambulatorial até 22.09 permanecendo negativo. As amostras coletadas foram denominadas $86 / 83$ e $95 / 83$.

$O$ terceiro paciente estudado (APJ), foi internado em 22.02.84 e tratado com a combina-

(*) De Souza. J. M. - Comunicação pessoal ção mefloquina $(0,75 \mathrm{~g})+$ sulfadoxina $(1,5 \mathrm{~g})$ + pirimetamina $(0,75 \mathrm{~g})$ em 23.02 , tendo vomitado 25 minutos após a ingestão da medicação, repetindo-se o vômito por mais duas vezes antes de completada a primeira hora. Tendo recrudescido em 16.03, foi-lhe aplicado tratamento único de mefloquina $(0,5 \mathrm{~g})$. Voltou a recrudescer em 07.04 sendo tratado em 09.04.84 com $1,0 \mathrm{~g}$ de mefloquina, curando definitivamente. As amostras coletadas foram denominadas: $19 / 84$ e $29 / 84$

Durante este periodo os pacientes permaneceram hospitalizados não havendo, portanto, quaisquer possibilidades de contatos com mosquitos infectados.

As amostras coletadas e cultivadas foram caracterizadas através das seguintes técnicas: testes de sensibilidade in vitro para cloroquina e mefloquina ${ }^{3}$ e tipificação enzimática para 2 enzimas: GPI - Glucose fosfato isomerase (EC. 5.3.1.9) e ADA - Adenosina deaminase (EC. 3.5.4.4) por eletroforese em acetato de celulose.

$\mathrm{Na}$ Tabela I estão sumarizadas as condições da corrida, tampões e reagentes utilizados.

As amostras submetidas à eletroforese foram comparadas sempre com amostras contro. les (cepa $\mathrm{k} 1$ e cepa $\mathrm{T} 94$ ) oriundas do Institute Animal Genetics, Edinburgh, Grã-Bretanha. Os testes de sensibilidade a cloroquina e mefloquina foram realizados em placas de 96 orifícios de fundo chato (Petécil, SP) com duração de 72 horas ${ }^{3}$. As diluições foram preparadas a partir de soluções estoque provenientes de OMS (Genebra, Suiça), tendo sido utilizadas as seguintes concentrações: $0 ; 1.0 ; 2.0 ; 4.0 ; 6.0$; $8.0 ; 16.0$ e $32.0 \times 10^{-8} \mathrm{M}$ para cloroquina e 0.5 ; $1.0 ; 2.0 ; 4.0 ; 6.0 ; 8.0$ e $16.0 \times 10^{-8} \mathrm{M}$ para mefloquina.

Com o objetivo de facilitar a leitura dos resultados observados na análise de cepas coletadas de pacientes recrudescentes, incluimos a Fig. 1 que corresponde a interpretação eletroforética para dois tipos enzimáticos de GPI e ADA.

\section{RESULTADOS E DISGUSSÃO}

Como se pode observar na Tabela II, o paciente FRI foi o que apresentou resultados mais surpreendentes, uma vez que cada recru- 
ROSARIO, V. E. do; COUTO, A. A.; SANTOS, M. A. \& SOUZA, J. M. de - Caracterização de cepas de P. falciparum coletadas de pacientes recrudescentes. Rev. Inst. Med. trop. São Paulo 27:274-278, 1985.

T A B E L A I

CONDICOEES DE ELETROFORESE

Componentes da reação de revelação

ENZ: GPI - Glucose-fosfato-isomerase

\section{Frutose-6-Phosphate (F-3627)}

$\mathrm{Mg} \quad \mathrm{C} 12$ (M-0250)

N A D P $(\mathrm{N}-0505)$

Glucose-6-Phosphate-Dehydrogenase (G-6378)

$M$ T T (M-2128)

$P$ M S (P-9625)

Tampão "TRIS HCL pH 8.0"

ENZ: ADA - Adenosina deaminase

Adenosine (A-9251)

Xanthine Oxidase ( $\mathrm{X}-1875)$

Nucleoside Phosphorilase (N-3003)

M T T (M-2128)

P M S (P-9625)

Tampão "Phosphate pH 7.5"

( ) - Referências SIGMA

Obs: As condiçōes descritas neste quadro são para placas de acetato de celulose de $76 \times 60 \mathrm{~mm}$.

$$
\begin{array}{r}
25 \mathrm{mg} \\
20 \mathrm{mg} \\
5 \mathrm{mg} \\
0,01 \mathrm{ml} \\
5 \mathrm{mg} \\
2 \mathrm{mg} \\
2 \mathrm{ml}
\end{array}
$$

$10 \mathrm{mg}$

$0,01 \mathrm{ml}$

$0,01 \mathrm{ml}$

$5 \mathrm{mg}$

$2 \mathrm{mg}$

$2 \mathrm{ml}$

ELETROFORESE EM ACETATO DE CELULOSE

CONDIÇÖES DA CORRIDA

Tempo: Voltagem: Miliamperagem:

$20 \mathrm{~min} \quad 160 \mathrm{~V} \quad 2$ a $3 \mathrm{~mA}$

TAMPOES

Enzima: Tanque Revelação

G P I SUPRE HEME TRIS HCl pH 8.0

A D A SUPRE HEME FOSFATO $\mathrm{pH} \quad 7.5$
CORRIDA PARA GPI

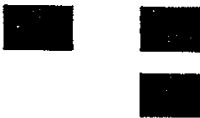

A

CORRIDA PARA ADA

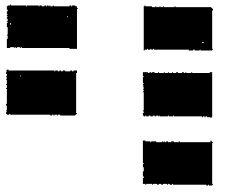

RESULTADO E LEITURA

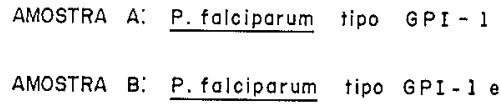

AMOSTRA A: P. falciparum tipo ADA - 1

AMOSTRA B: P. falciporum tipo ADA-1 a ADA - 2 (Populaçāo mista)

CONCLUSÄO: A amostra A é constituído por parasitas identicos quanto cos enzimas GPI e ADA, ou seja, todos sendo do tipo GPI - 1, ADA-1. A amostra $B \dot{E}$ constituída por uma mistura de parasitas, podendo ser misturos de 2 ou meis tipos, como: GPI-1, ADA-1; GPI-I, ADA - 2; GPI - 2, ADA-1 e possivelmente também GPI-2, ADA-2.

Fig. 1 - Interpretação de resultados obtidos pela técnica de eletroforese

descência exibiu um tipo enzimático distinto ou seja, uma nova população de P. falciparum. Dado que o paciente esteve sempre internado, nãc houve qualquer possibilidade de reinfeç̧ão e, portanto, podemos supor que a população de P. falciparum do tipo GPI-2/ADA-2 apenas revelada na $3^{\text {a }}$ recrudescência (cepa $97 / 83$ ) estava presente desde o início da infecção, inclusive na cepa original 70/83. Lamentavelmente a detecção de uma determinada enzima pela técnica de eletroforese, quer em acetato de celulose, quer em gel de amido, requer parasitemias elevadas mesmo em cultivo; por esse motivo cremos não ter sido possivel detectar estes tipos enzimá. ticos nas recrudescências anteriores.

O paciente (PPS) correspondente às cepas 86 e $95 / 83$ não apresentou diferenças nos tipos 

ROSARIO, V. E. do; COUTO, A. A.; SANTOS, M. A. \& SOUZA, J. M. de - Caracterização de cepas de P. falciparum co.
letadas de pacientes recrudescentes. Rev. Inst. Med. trop. São Paulo 27:274-278, 1985,

T A B E L A II

Tipo enzimático e nivel de sensibilidade a drogas em 3 pacientes analisados

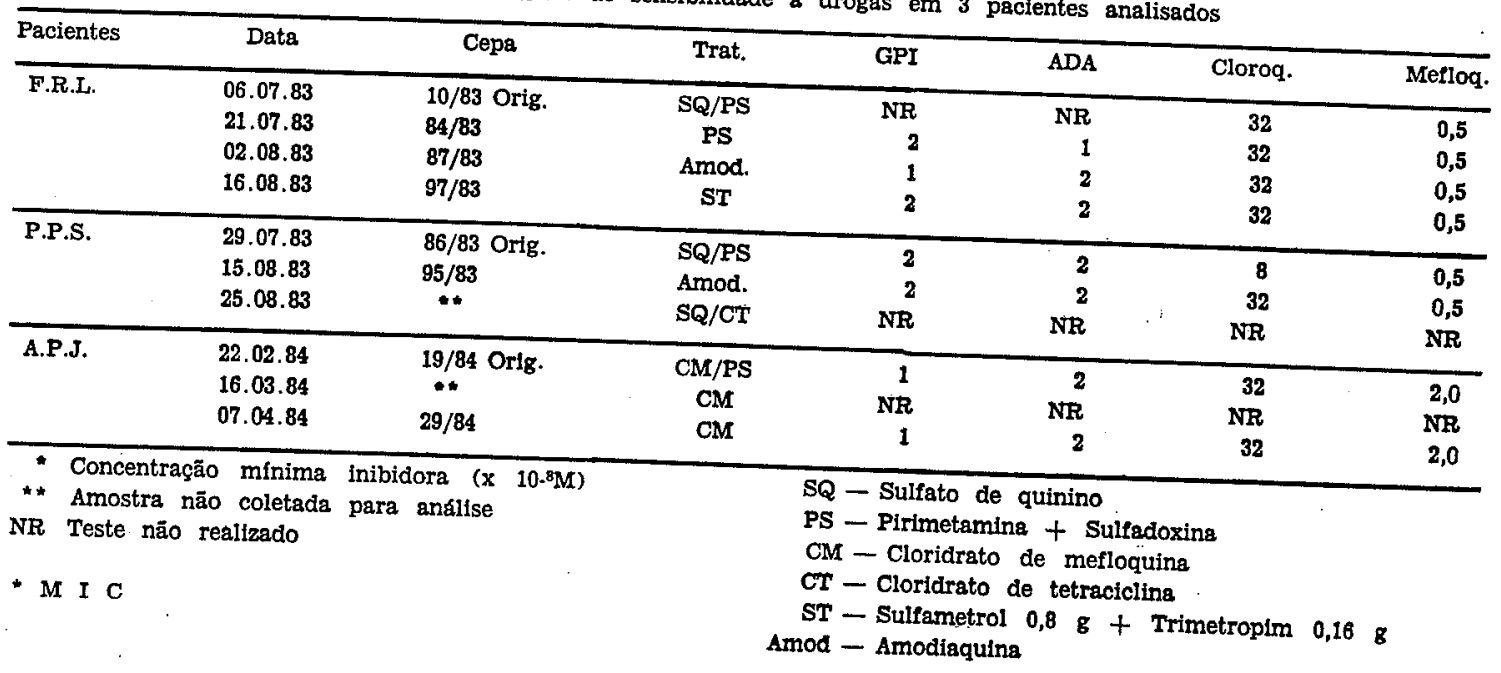

enzimáticos analisados entre as cepas original e recrudescente. Entretanto, verificou-se uma alteração no nível de sensibilidade para cloroquina, onde na cepa original a concentração minima inibidora (MIC) foi $8 \times 10^{-8} \mathrm{M}$, e na amostra correspondente à $2{ }^{a}$ recrudescência foi $32 \mathrm{x}$ $10^{-8} \mathrm{M}$, apesar do paciente não ter sido tratado em nenhuma ocasião com a mencionada droga. Portanto, a medicação com sulfato de quinino! pirimetamina-sulfadoxina selecionou uma população resistente à cloroquina. Isto sugere que na população original já se encontravam parasitos com maior resistência, porém, em menor pro. porção. Com a ação da associação sulfato de quinino/pirimetamina-sulfadoxina, houve a eliminação de grande parte dos parasitos, havendo, entretanto, o crescimento dos mais resistentes, inclusive à cloroquina.

O terceiro paciente recrudescente estudado neste trabalho (APJ) correspondente às cepas 19/84 e 29/84, também não apresentou altera. ções em seus tipos enzimáticos, assim como não apresentou modificação no nível de sensibili. dade para os testes realizados.

Isto é um exemplo interessante de uma recrudescência tardia motivada talvez pela subdosagem de mefloquina (devido a vômito) dado que a dose global única curou este paciente. Daí se pode presumir que combinações tera. pêuticas serão efetivas se ambos os medicamen. tos tiverem ação sobre a população de parasitas.
Apesar deste estudo ter sido feito tomando como ponto de referência o perfil enzimático $\mathrm{e}$ a sensibilidade à drogas, deve-se salientar que não há qualquer relação comprovada entre tipo enzimático e sensibilidade a drogas, dado que os genes que regulam a resistência e os responsáveis pelas formas enzimáticas não demonstraram até o momento prova de ligação gênica 5 . Entretanto, estes métodos são eficazes na caracterização precisa dos vários tipos de cepas de $\mathbf{P}$. falciparum ocorrentes em um único paciente.

Acreditamos que o estudo de um maior número de cepas de pacientes recrudescentes seja necessário para proporcionar melhores con. clusões sobre esta matéria e que se deva acentuar a importância da pesquisa em casos clíni$\cos$ de pacientes recrudescentes, que darão uma melhor compreensão ao problema de resistên. cia e dinâmica de subpopulaçôes em um mesmo paciente.

\section{SUMMARY}

Characterization of $P$. falciparum strains obtained from recrudescent patients

Characterization of Plasmodium falciparum strains obtained from three patients interned in the Clinico-Pharmalogical Testing Centre of the Barros Barreto Hospital, Belém, Pará, Brazil, was carried out using cellulose acetate elec. trophoresis for the two enzymes glucose phosphate isomerase EC.5.3.1.9 (GPI) and adenosine diaminase EC.3.5.4.4 (ADA) and sensibility 
ROSARIO, V. E. do; COUTO, A. A.; SANTOS, M. A. \& SOUZA, J. M. de - Caracterizaçăo de cepas de P. falciparum coletadas de pacientes recrudescentes. Rev. Inst. Med. trop. São Paulo 27:274-278, 1985.

tests for the two antimalarial drugs, chloroquin and mefloquine. This characterization showed an enzyme variant in one of the patients, and a modification in the chloroquine ser.sibility level in another; not only in the strains from the original infections, but also in those from each of the relapses.

\section{AGRADECIMENTOS}

Este trabalho foi realizado com o apoio da Fundação SESP/M.S., CNPq, FINEP , HBB/ SUCAM e com o auxilio da UNDP/WORLD BANK/WHO Special Programme for Research and Training in Tropical Diseases. Agradecemos, também, à Dra. Salma Oliveira pelo apoio técnico prestado; à Dra. Fátima Assis pela revisão do manuscrito e a Sra. Margarete Garcia pela elaboração das tabelas.

\section{REFERENCIAS BIBLIOGRAFICAS}

1 COUTO, A. A.; ROSARIO, V. E. \& WALLIKER, D. Análise enzimática de 56 amostras de $P$. falciparum da Bacia Amazónica. Rev. Bras. Malariol. Doenças Trop. 35: 11-19, 1983.

2. ROSARIO; V. E. - Caracterização de cepas de P. falciparum do Brasil. Rev. FSESP 28: 115-136, 1983.

3. SANTOS, M. V. \& ROSARIO, V. E. - Testes de sensbilidade in vitro de amostras de $\mathbf{P}$. talclparum da Bacia Amazónica. Rev. Bras. Malartol. Doenças Trop. 35: $18-21,1983$.

4. SOUZA, J. M. - A phase II clinical trial of Mefloquine in Brazilian male subjects. Bull. WHO 61: 815-820, 1983.

5. THAITHONG, S. - Biological characterization of malaria parasites by means of enzyme identification on pattern analysis. WHO/MAL 935: 1-6, 1981.

6. TRAGER, W. \& JENSEN, J. B. - Human malaria parasites in continuous culture. Seience 193: 674-675, 1976.

Recebido para publicaçāo em 30/11/1984. 